\title{
Determination of the absolute branching fractions of $X(3872)$ decays
}

\author{
Chunhua $\mathrm{Li}^{1, *}$ and Chang-Zheng Yuan $\odot^{2,3, \dagger}$ \\ ${ }^{1}$ School of Physics and Electronic Technology, Liaoning Normal University, Dalian 116029, China \\ ${ }^{2}$ Institute of High Energy Physics, Chinese Academy of Sciences, Beijing 100049, China \\ ${ }^{3}$ University of Chinese Academy of Sciences, Beijing 100049, China
}

(Received 23 July 2019; published 5 November 2019)

\begin{abstract}
We report the first determination of the absolute branching fractions of the $X(3872)$ decays by globally analyzing the measurements provided by the Belle, BABAR, BESIII, and LHCb experiments. The branching fractions of $X(3872) \rightarrow \pi^{+} \pi^{-} J / \psi$ and $D^{* 0} \bar{D}^{0}+$ c.c. are found to be $\left(4.1_{-1.1}^{+1.9}\right) \%$ and $\left(52.4_{-14.3}^{+25.3}\right) \%$, respectively. The branching fractions of the decays $X(3872) \rightarrow \gamma J / \psi, \gamma \psi(3686), \omega J / \psi$, and $\pi^{0} \chi_{c 1}$ are also determined. The global fit implies that the fraction of $X(3872)$ decays which are not observed in experiments is $\left(31.9_{-31.5}^{+18.1}\right) \%$, which indicates that there is still a lot of room for searching for new decay modes of the $X(3872)$. With the branching fraction, we determine the production cross section of $e^{+} e^{-} \rightarrow \gamma X(3872)$ at center-of-mass energy $4.226 \mathrm{GeV}$.
\end{abstract}

DOI: $10.1103 /$ PhysRevD.100.094003

Observed in 2003 by the Belle experiment [1], the $X(3872)$ has been the most puzzling state among all the so-called charmoniumlike XYZ states for all these years [2]. Being very close to the $D^{* 0} \bar{D}^{0}+$ c.c. mass threshold, it is supposed to have a large $D^{* 0} \bar{D}^{0}+$ c.c. molecule component [3], and its large production rate in $p p$ and $p \bar{p}$ collision experiments suggests that there is a charmonium state $\chi_{c 1}(2 P)$ core in its wave function [4-7]. The absolute branching fractions of observed and unknown decays of $X(3872)$ could provide essential information to distinguish the different scenarios [8-11].

During the last 16 years, there have been many experimental measurements on the $X(3872)$; however, its mass was measured in a high precision of $170 \mathrm{keV}$ [12] and its spin-parity and charge-conjugate parity quantum numbers were determined to be $J^{P C}=1^{++}$[13], all other quantities are only known with very large uncertainties. Its total width is known to be an upper limit of $1.2 \mathrm{MeV}$ at the $90 \%$ confidence level (C.L.), and only $D^{* 0} \bar{D}^{0}+$ c.c., $\pi^{+} \pi^{-} J / \psi$, $\omega J / \psi, \gamma J / \psi$, and $\pi^{0} \chi_{c 1}$ decay modes are observed with more than 5 standard deviation significance [14-23], and the existence of the $\gamma \psi(3686)$ decay mode is not confirmed [19,22-24]. None of the above modes have measured absolute branching fractions due to the fact that the total number of $X(3872)$ events is very difficult to be determined

\footnotetext{
*hunhua@lnnu.edu.cn

†yuancz@ihep.ac.cn
}

Published by the American Physical Society under the terms of the Creative Commons Attribution 4.0 International license. Further distribution of this work must maintain attribution to the author(s) and the published article's title, journal citation, and DOI. Funded by SCOAP ${ }^{3}$. either in $B$-meson decays or in an $e^{+} e^{-}$annihilation experiment. As a consequence, only the product branching fractions in $B$ decays or the product of the total cross section and the $X(3872)$ decay branching fraction is reported experimentally.

$B A B A R$ and Belle experiments tried to measure $\mathcal{B}\left(B^{+} \rightarrow X(3872) K^{+}\right)$by reconstructing inclusive $B^{+} \rightarrow$ $K^{+}+$anything and searching for the monochromatic $K^{+}$ corresponds to two-body decay $B^{+} \rightarrow X(3872) K^{+}$in the center-of-mass system of the $B^{+}$-meson $[25,26]$, but no significant signals were observed. By improving the algorithm of signal reconstruction and using the full available data, $B A B A R$ is able to improve the sensitivity of the inclusive $X(3872)$ reconstruction, a $3 \sigma$ significance signal of $B^{+} \rightarrow X(3872) K^{+}$is observed, and the branching fraction is determined to be $(2.1 \pm 0.6 \pm 0.3 \pm 0.1) \times$ $10^{-4}$ [27], where the three uncertainties are statistical, systematic, and in the quoted $\mathcal{B}\left(B^{+} \rightarrow J / \psi K^{+}\right)$, respectively. Belle found only a $1.1 \sigma X(3872)$ signal, and provided an upper limit $\mathcal{B}\left(B^{+} \rightarrow X(3872) K^{+}\right)<2.6 \times 10^{-4}$ at the $90 \%$ C.L., and a central value of $(1.2 \pm 1.1 \pm$ $0.1) \times 10^{-4}$, where the uncertainties are statistical and systematic, respectively.

Although neither the BABAR nor the Belle measurement is significant enough to claim observation of the $B^{+} \rightarrow$ $X(3872) K^{+}$signal, we do know this decay exists since significant signals have been observed when $X(3872)$ is reconstructed in its exclusive decay modes such as $\pi^{+} \pi^{-} J / \psi$ and $D^{* 0} \bar{D}^{0}+$ c.c. This allows us to extract the $X(3872)$ decay branching fractions by using the $B A B A R$ and Belle measurements of $\mathcal{B}\left(B^{+} \rightarrow X(3872) K^{+}\right)$and the other measurements from Belle, BABAR, BESIII, and $\mathrm{LHCb}$ experiments. 
The measurements used in this analysis are listed in Table I. To extract the absolute branching fractions of $X(3872)$ decays we do a least square fit by minimizing

$$
\chi^{2}(x)=\sum_{i=1}^{25} \frac{\left(x_{i}-x\right)^{2}}{\sigma_{i}^{2}},
$$

where $i$ is the index (from 1 to 25) listed in Table I, $x_{i}$ are the 25 measured values, $x$ is the corresponding values

TABLE I. The measurements of the $X(3872)$ decays by Belle, $B A B A R$, BESIII, and $\mathrm{LHCb}$ experiments: the product branching fractions of $X(3872) \rightarrow \pi^{+} \pi^{-} J / \psi, \gamma J / \psi, \gamma \psi(3686), D^{* 0} \bar{D}^{0}+$ c.c., and $\omega J / \psi$, the inclusive branching fraction $\mathcal{B}(B \rightarrow X(3872) K)$, the ratios of the branching fractions $\mathcal{B}(X(3872) \rightarrow \gamma J / \psi$, $\omega J / \psi, D^{* 0} \bar{D}^{0}+$ c.c.,$\left.\pi^{0} \chi_{c 1}\right)$ to $\mathcal{B}\left(X(3872) \rightarrow \pi^{+} \pi^{-} J / \psi\right)$. The first uncertainties are statistical and the second systematic.

\begin{tabular}{|c|c|c|c|}
\hline Index $(i)$ & Parameters & Values & Experiments \\
\hline & \multicolumn{3}{|c|}{$X(3872) \rightarrow \pi^{+} \pi^{-} J / \psi\left(\times 10^{-6}\right)$} \\
\hline 1 & \multirow[t]{2}{*}{$B^{+} \rightarrow X(3872) K^{+}$} & $8.61 \pm 0.82 \pm 0.52$ & Belle [14] \\
\hline 2 & & $8.4 \pm 1.5 \pm 0.7$ & $B A B A R$ [15] \\
\hline 3 & \multirow[t]{2}{*}{$B^{0} \rightarrow X(3872) K^{0}$} & $4.3 \pm 1.2 \pm 0.4$ & Belle [14] \\
\hline 4 & & $3.5 \pm 1.9 \pm 0.4$ & $B A B A R[15]$ \\
\hline & \multirow{3}{*}{$\begin{array}{l}X(3872) \rightarrow \gamma J / \psi \\
B^{+} \rightarrow X(3872) K^{+}\end{array}$} & $\left(\times 10^{-6}\right)$ & \\
\hline 5 & & $1.78_{-0.44}^{+0.48} \pm 0.12$ & Belle [22] \\
\hline 6 & & $2.8 \pm 0.8 \pm 0.1$ & $B A B A R$ [23] \\
\hline 7 & \multirow{2}{*}{$B^{0} \rightarrow X(3872) K^{0}$} & $1.24_{-0.61}^{+0.76} \pm 0.11$ & Belle [22] \\
\hline 8 & & $2.6 \pm 1.8 \pm 0.2$ & $B A B A R[23]$ \\
\hline \multirow{6}{*}{$\begin{array}{l}9 \\
10 \\
11 \\
12\end{array}$} & \multicolumn{3}{|c|}{$X(3872) \rightarrow \gamma \psi(3686)\left(\times 10^{-6}\right)$} \\
\hline & $B^{+} \rightarrow X(3872) K^{+}$ & $0.83_{-1.83}^{+1.98} \pm 0.44$ & Belle [22] \\
\hline & \multirow{3}{*}{$B^{0} \rightarrow X(3872) K^{0}$} & $9.5 \pm 2.7 \pm 0.6$ & $B A B A R$ [23] \\
\hline & & $1.12_{-2.90}^{+3.57} \pm 0.57$ & Belle [22] \\
\hline & & $11.4 \pm 5.5 \pm 1.0$ & $B A B A R$ [23] \\
\hline & $\begin{array}{l}X(3872) \rightarrow D^{* 0} \bar{D}^{0} \\
\quad+\text { c.c. }\end{array}$ & \multicolumn{2}{|l|}{$\left(\times 10^{-4}\right)$} \\
\hline 13 & \multirow[t]{2}{*}{$B^{+} \rightarrow X(3872) K^{+}$} & $0.77 \pm 0.16 \pm 0.10$ & Belle [16] \\
\hline 14 & & $1.67 \pm 0.36 \pm 0.47$ & $B A B A R$ [17] \\
\hline 15 & \multirow{2}{*}{$B^{0} \rightarrow X(3872) K^{0}$} & $0.97 \pm 0.46 \pm 0.13$ & Belle [16] \\
\hline \multirow[t]{2}{*}{16} & & $2.22 \pm 1.05 \pm 0.42$ & $B A B A R[17]$ \\
\hline & \multirow{3}{*}{$\begin{array}{l}X(3872) \rightarrow \omega J / \psi \\
B^{+} \rightarrow X(3872) K^{+} \\
B^{0} \rightarrow X(3872) K^{0}\end{array}$} & $\left(\times 10^{-6}\right)$ & \\
\hline 17 & & $6 \pm 2 \pm 1$ & $B A B A R[18]$ \\
\hline 18 & & $6 \pm 3 \pm 1$ & $B A B A R[18]$ \\
\hline 19 & \multirow{5}{*}{ 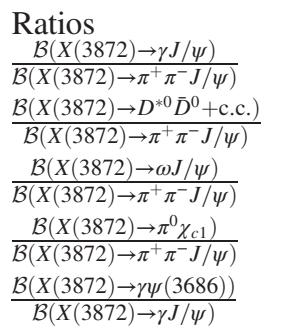 } & $0.79 \pm 0.28$ & BESIII [19] \\
\hline 20 & & $14.81 \pm 3.80$ & BESIII [19] \\
\hline 21 & & $1.6_{-0.3}^{+0.4} \pm 0.2$ & BESIII [20] \\
\hline 22 & & $0.88_{-0.27}^{+0.33} \pm 0.10$ & BESIII [21] \\
\hline \multirow[t]{2}{*}{23} & & $2.46 \pm 0.64 \pm 0.29$ & LHCb [24] \\
\hline & \multirow[t]{3}{*}{$B^{+} \rightarrow X(3872) K^{+}$} & $\left(\times 10^{-4}\right)$ & \\
\hline 24 & & $2.1 \pm 0.6 \pm 0.3$ & $B A B A R$ [27] \\
\hline 25 & & $1.2 \pm 1.1 \pm 0.1$ & Belle [26] \\
\hline
\end{tabular}

constructed with free parameters, and $\sigma_{i}$ is the sum of the statistical and systematic uncertainties in quadrature.

In the above definition, we assumed that all the measurements follow Gaussian distribution, the statistical uncertainties of different measurements are uncorrelated, and possible correlation among the systematic uncertainties of different measurements in an experiment is neglected since the statistical uncertainties are dominant for most of the measurements. We also assume that there is no correlation between different experiments.

The branching fractions $\mathcal{B}\left(X(3872) \rightarrow \pi^{+} \pi^{-} J / \psi\right)$, $\mathcal{B}\left(X(3872) \rightarrow D^{* 0} \bar{D}^{0}+\right.$ c.c. $), \quad \mathcal{B}(X(3872) \rightarrow \gamma J / \psi)$, $\mathcal{B}(X(3872) \rightarrow \gamma \psi(3686)), \quad \mathcal{B}(X(3872) \rightarrow \omega J / \psi)$, $\mathcal{B}\left(X(3872) \rightarrow \pi^{0} \chi_{c 1}\right), \mathcal{B}\left(B^{+} \rightarrow X(3872) K^{+}\right)$, and $\mathcal{B}\left(B^{0} \rightarrow\right.$ $\left.X(3872) K^{0}\right)$ are free parameters in the fit. By minimizing $\chi^{2}(x)$ with MINUIT [28], the fitting results are obtained and listed in Table II. The fit yields $\chi^{2} / \mathrm{ndf}=25.2 / 17$ where ndf represents the number of degrees of freedom. The correlation coefficients between the fit parameters are shown in Table III.

From the fitting results we can see that $D^{* 0} \bar{D}^{0}+$ c.c. is the dominant decay mode of the $X(3872)$ with a decay rate of $\left(52.4_{-14.3}^{+25.3}\right) \%$, and all the other modes with charmonium have branching fraction of a few percent. With all of the known branching fractions, the fraction of $X(3872)$ decays which is not yet observed in experiment is determined to be $\left(31.9_{-31.5}^{+18.1}\right) \%$, so the search for new decay modes of the $X(3872)$ is still an important task. As free parameters in the fitting, the production rates of the $X(3872)$ in neutral and charged $B$-meson decays are obtained as listed in Table II.

BESIII measured the product $\sigma\left(e^{+} e^{-} \rightarrow \gamma X(3872)\right) \times$ $\mathcal{B}\left(X(3872) \rightarrow \pi^{+} \pi^{-} J / \psi\right)$ at center-of-mass energies from 4 to $4.6 \mathrm{GeV}$ [20]; with known $\mathcal{B}\left(X(3872) \rightarrow \pi^{+} \pi^{-} J / \psi\right)$, the production cross section of $e^{+} e^{-} \rightarrow \gamma X(3872)$ can be obtained. The peak cross section of $e^{+} e^{-} \rightarrow \gamma X(3872)$ is found to be $\left(5.5_{-3.6}^{+2.8}\right) \mathrm{pb}$ at the center-of-mass energy $4.226 \mathrm{GeV}$. This is obtained by sampling both numerator and denominator with the consideration of their asymmetric

TABLE II. The fitting results of the absolute branching fractions of the $X(3872)$ decays and $B \rightarrow X(3872) K$ decays. The branching fraction of $X(3872)$ decays into unknown modes is calculated from the fit results.

\begin{tabular}{lll}
\hline \hline Parameter index & \multicolumn{1}{c}{ Decay mode } & Branching fraction \\
\hline 1 & $X(3872) \rightarrow \pi^{+} \pi^{-} J / \psi$ & $\left(4.1_{-1.1}^{+1.9}\right) \%$ \\
2 & $X(3872) \rightarrow D^{* 0} \bar{D}^{0}+$ c.c. & $\left(52.4_{-14.3}^{+25.3}\right) \%$ \\
3 & $X(3872) \rightarrow \gamma J / \psi$ & $\left(1.1_{-0.3}^{+0.6}\right) \%$ \\
4 & $X(3872) \rightarrow \gamma \psi(3686)$ & $\left(2.4_{-0.8}^{+1.3}\right) \%$ \\
5 & $X(3872) \rightarrow \pi^{0} \chi_{c 1}$ & $\left(3.6_{-1.6}^{+2.2}\right) \%$ \\
6 & $X(3872) \rightarrow \omega J / \psi$ & $\left(4.4_{-1.3}^{+2.3}\right) \%$ \\
7 & $B^{+} \rightarrow X(3872) K^{+}$ & $(1.9 \pm 0.6) \times 10^{-4}$ \\
8 & $B^{0} \rightarrow X(3872) K^{0}$ & $\left(1.1_{-0.4}^{+0.5}\right) \times 10^{-4}$ \\
& $X(3872) \rightarrow$ unknown & $\left(31.9_{-31.5}^{+18.1}\right) \%$ \\
\hline \hline
\end{tabular}


TABLE III. Correlation coefficients of the fit parameters listed in Table II.

\begin{tabular}{lcccccccc}
\hline \hline Parameter index & 1 & 2 & 3 & 4 & 5 & 6 & 7 & 8 \\
\hline 1 & 1 & 0.87 & 0.84 & 0.75 & 0.64 & 0.79 & -0.95 & -0.87 \\
2 & & 1 & 0.79 & 0.71 & 0.56 & 0.74 & -0.90 & -0.77 \\
3 & & & 1 & 0.78 & 0.54 & 0.73 & -0.88 & -0.78 \\
4 & & & & 1 & 0.49 & 0.65 & -0.79 & -0.69 \\
5 & & & & & 1 & 0.51 & -0.61 & -0.56 \\
6 & & & & & & 1 & -0.82 & -0.72 \\
7 & & & & & & & 1 & 0.84 \\
\hline \hline
\end{tabular}

uncertainties; the cross section is about an order of magnitude smaller than that of $e^{+} e^{-} \rightarrow \pi^{+} \pi^{-} J / \psi$ at the same energy [29].

In summary, we obtain the absolute branching fractions of the $X(3872)$ decays into six modes by globally fitting the measurements provided by Belle, BABAR, BESIII, and LHCb experiments. The branching fraction of $X(3872) \rightarrow$ $\pi^{+} \pi^{-} J / \psi$ is determined to be $\left(4.1_{-1.1}^{+1.9}\right) \%$ which can be used in many measurements where $X(3872) \rightarrow \pi^{+} \pi^{-} J / \psi$ is reconstructed and can supply critical input in understanding the nature of the $X(3872)$ [30]. This branching fraction is in good agreement with early estimations in Refs. [31] and [32]. By combining the branching fractions of the observed modes, we obtain the fraction of the unknown decays of the $X(3872)$ as $\left(31.9_{-31.5}^{+18.1}\right) \%$, which calls for more experimental efforts in the study of the $X(3872)$ decays.
The results were first presented at the Workshop "Exotic Hadrons: Theory and Experiment at Lepton and Hadron Colliders," the authors thank the organizers, Prof. Luciano Maiani and Prof. Wei Wang, for their hospitality. This work is supported in part by LiaoNing Revitalization Talents Program No. XLYC1807135; Scientific Research Foundation of LiaoNing Normal University No. BS2018L005; National Natural Science Foundation of China (NSFC) under Contracts No. 11835012 and No. 11521505; Key Research Program of Frontier Sciences, CAS, Grant No. QYZDJ-SSW-SLH011; and the CAS Center for Excellence in Particle Physics (CCEPP).

Note added.-After this paper was submitted, a paper titled "Branching Fractions of the $X(3872)$ " by Braaten, He, and Ingles [33] appeared. Instead of treating the $X(3872)$ as a single structure which has universal properties in different production and decay mechanisms as in our paper, these authors considered possible contributions of $D^{* 0} \bar{D}^{0}+$ c.c. threshold effect and a bound state below the threshold or a virtual state in $B^{+} \rightarrow K^{+} X(3872)$ decay, and reported estimations of the branching fractions of a bound state of charm mesons [part of the $X(3872)$ structure]. Future measurements of the line shapes of the $X(3872)$ structures in $B^{+} \rightarrow K^{+} X(3872), B^{0} \rightarrow K^{0} X(3872)$, and $e^{+} e^{-} \rightarrow$ $\gamma X(3872)$ may check these above assumptions and reveal the nature of the $X(3872)$.
[1] S. K. Choi et al. (Belle Collaboration), Phys. Rev. Lett. 91, 262001 (2003).

[2] For a recent review, see N. Brambilla, S. Eidelman, C. Hanhart, A. Nefediev, C. P. Shen, C. E. Thomas, A. Vairo, and C. Z. Yuan, arXiv:1907.07583.

[3] E. S. Swanson, Phys. Lett. B 598, 197 (2004); Phys. Rep. 429, 243 (2006).

[4] R. Aaij et al. (LHCb Collaboration), Eur. Phys. J. C 72, 1972 (2012).

[5] S. Chatrchyan et al. (CMS Collaboration), J. High Energy Phys. 04 (2013) 154.

[6] M. Aaboud et al. (ATLAS Collaboration), J. High Energy Phys. 01 (2017) 117.

[7] V. M. Abazov et al. (D0 Collaboration), Phys. Rev. Lett. 93, 162002 (2004).

[8] J. Ferretti, G. Galatà, and E. Santopinto, Phys. Rev. D 90, 054010 (2014).

[9] E. J. Eichten, K. Lane, and C. Quigg, Phys. Rev. D 73, 014014 (2006).

[10] N. N. Achasov and E. V. Rogozina, JETP Lett. 100, 227 (2014).

[11] X. W. Kang and J. A. Oller, Eur. Phys. J. C 77, 399 (2017).
[12] M. Tanabashi et al. (Particle Data Group), Phys. Rev. D 98, 030001 (2018).

[13] R. Aaij et al. (LHCb Collaboration), Phys. Rev. Lett. 110, 222001 (2013).

[14] S.-K. Choi et al. (Belle Collaboration), Phys. Rev. D 84, 052004 (2011).

[15] B. Aubert et al. (BABAR Collaboration), Phys. Rev. D 77, 011102 (2008).

[16] T. Aushev et al. (Belle Collaboration), Phys. Rev. D 81, 031103 (2010).

[17] B. Aubert et al. (BABAR Collaboration), Phys. Rev. D 77, 011102 (2008).

[18] P. del Amo Sanchez et al. (BABAR Collaboration), Phys. Rev. D 82, 011101 (2010).

[19] J. H. Yin (BESIII COllaboration), Recent results on the X (3872) at BESIII, QWG 2019, Torino, Italy (2019), https:// agenda.infn.it/event/15632/contributions/89304/ attachments/63001/75737/

Recent_result_of_X3872_on_BESIII.pdf.

[20] M. Ablikim et al. (BESIII Collaboration), Phys. Rev. Lett. 122, 232002 (2019).

[21] M. Ablikim et al. (BESIII Collaboration), Phys. Rev. Lett. 122, 202001 (2019). 
[22] V. Bhardwaj et al. (Belle Collaboration), Phys. Rev. Lett. 107, 091803 (2011).

[23] B. Aubert et al. (BABAR Collaboration), Phys. Rev. Lett. 102, 132001 (2009).

[24] R. Aaij et al. (LHCb Collaboration), Nucl. Phys. B886, 665 (2014).

[25] B. Aubert et al. (BABAR Collaboration), Phys. Rev. Lett. 96, 052002 (2006).

[26] Y. Kato et al. (Belle Collaboration), Phys. Rev. D 97, 012005 (2018).

[27] G. Wormser (BABAR Collaboration), Charmonium(-like) decays at BABAR, QWG 2019, Torino, Italy (2019), https:// agenda.infn.it/event/15632/contributions/89320/ attachments/63025/75778/charmonium_QWG19.pdf.

[28] F. James and M. Roos, Comput. Phys. Commun. 10, 343 (1975).

[29] M. Ablikim et al. (BESIII Collaboration), Phys. Rev. Lett. 118, 092001 (2017).

[30] P. G. Ortega and E. R. Arriola, arXiv:1907.01441.

[31] C. Z. Yuan (Belle Collaboration), arXiv:0910.3138.

[32] A. Esposito, A. L. Guerrieri, F. Piccinini, A. Pilloni, and A. D. Polosa, Int. J. Mod. Phys. A 30, 1530002 (2015).

[33] E. Braaten, L. P. He, and K. Ingles, arXiv:1908.02807. 